\title{
Transient Stuttering in Catatonic Bipolar Patients
}

\author{
ANTHONY B. JOSEPH
}

The Center for Neurobehavioral Rehabilitation, Greenery Rehabilitation Group; Psychopharmacology Program, McLean Hospital, Department of Psychiatry, Harvard Medical School, Boston MA, USA

\begin{abstract}
Two cases of transient stuttering occurring in association with catatonia and bipolar disorder are described. Affective decompensation has been associated with lateralized cerebral dysfunction, and it is hypothesized that in some bipolar catatonic patients a concomitant disorder of the lateralization of language function may lead to a variety of clinical presentations including aphasia, mutism, and stuttering.
\end{abstract}

\section{Introduction}

Considered a subtype of schizophrenia in the past, catatonia is now known to be more commonly associated with affective disorders, temporal lobe epilepsy, and cerebellar dysfunction (Gelenberg, 1976; Joseph et al., 1985; Slater and Roth, 1977).

Two cases are reported in which stuttering occurred with both catatonia and bipolar affective disorder. The neuropsychiatric findings in these patients are discussed with reference to theories of cerebral dominance, laterality and language.

\section{Case 1}

$\mathrm{Mr}$ A a right-handed 50-year-old, single white male, was well until the onset of depression 1 week before admission to hospital. His psychiatric history was positive for bipolar affective disorder. On two previous occasions he had become catatonic when depressed. His medical history was negative. There was no family history of psychiatric or neurologic disease.

The day before admission he became akinetic, mute, and cataleptic. On admission, he was catatonic, psychotic and depressed. Neurological examination revealed a fine rhythmical tremor of his hands and feet. When placed in a sitting, standing or lying position, he would remain in it. He was almost completely unable to obey even the simplest motor commands, except occasionally in an incomplete and perserverative manner. He was virtually mute, with only occasional sparse, telegraphic responses to questions. Comprehension and rep- 
etition were intact. There was no nominal aphasia or difficulty with color naming. Reading and writing appeared to be preserved although formal testing was difficult. He had a number of complex motor and mental status abnormalities in addition to paranoia and depression. These included: impaired eye tracking; lateral gaze impersistence; cogwheeling; gegenhalten; a positive glabellar response; positive suck and grasp reflexes; an occulocephalic reflex; inability to remember any of three objects; disorientation for date; and catalepsy.

In summary, he was psychotic, catatonic, and depressed, while showing profound abnormalities of the motor system and multiple pathological signs and reflexes usually associated with diffuse cerebral dysfunction such as dementia or delirium (Jenkyn et al., 1977). He also had a profound language disorder, almost approaching complete mutism.

Mr A improved with benzodiazepines and lithium carbonate, followed by a course of ECT. As the catatonia began to resolve, the language disorder became fluent with impaired comprehension and preserved repetition. The motor examination returned to normal except for a prominent residual occulocephalic reflex. The language disorder then resolved completely to be replaced by 2 days of stuttering. The occulocephalic reflex was the last of the neurological and mental status findings to resolve, and disappeared by the 17th day of his admission.

Routine laboratory evaluation had been unremarkable. Computed tomography revealed frontal lobe and brainstem atrophy with enlargement of the Sylvian and interhemispheric fissures. No independent cause for this could be determined and it was concluded that it was idiopathic atrophy in association with bipolar affective disorder.

Mr A was discharged in remission on maintenance lithium carbonate.

\section{Case 2}

Ms B a 49-year-old married white female, was admitted for evaluation of catatonia. Her medical history was negative, except for affective disorder and past alcohol abuse.

She had become non-compliant with medication in the context of a major depressive episode 1 week before admission. After several days the following phenomena occurred against a background of increasing catatonia: she stuttered for a whole day; the next day she had marked clonic shaking of her limbs, particularly the right forearm; subsequently she was found in the foetal position, unresponsive, and neither talking nor moving; a relative was unable to straighten her legs because "they were locked tight". Later she was taken to an emergency room and hospitalized. During this period, she was described as "dazed"; her eyes were said to be open and "glassy", and she was unresponsive. She was amnestic for events between the end of her stuttering and several hours after her admission to the hospital.

On admission, she was diagnosed as having bipolar depression and resolving catatonia. Physical examination revealed a rhythmical resting tremor in the arms, right more than left, moderate gegenhalten and cogwheeling, strongly positive 
suck and bilateral grasp reflexes and utilization behavior as described by Lhermitte (1983).

No aphasia was noted, although the history was consistent with an immediately previous transient, expressive, non-fluent disorder of language progressing to almost complete mutism on admission.

Her neurological signs fluctuated rapidly throughout the admission. Within a day, a strongly positive glabellar response had developed which could even be elicited by tapping the vertex or occiput; the following day the tremor, cogwheeling and utilization behavior were much improved, but the glabellar response was unchanged. The general trend was for improvement of her neurological status, but on some days it worsened, and new signs would appear or disappear. Thirteen days after admission, her depression, catatonia, and all neurological abnormalities, except the glabellar response, had disappeared.

Ms B denied any previous stuttering, but her two right-handed sons stutter. One also has an unspecified learning disability. She has mixed dominance for handedness. With her right hand, she writes on a blackboard, with her left on paper; with her right hand she deals cards; with either hand she uses a key, shakes hands, combs her hair, brushes her teeth, or catches a ball.

Routine laboratory evaluation was unremarkable as was her electroencephalogram. Neuropsychological testing showed diffuse cortical dysfunction with particular impairment of the frontal lobes. Computed tomography revealed frontal, temporal, parietal, and vermian atrophy, with enlargement of the Sylvian and interhemispheric fissures. No cause for this could be established. It was concluded that the atrophy was either idiopathic in association with bipolar affective disorder, secondary to her past alcohol abuse, or a combination of both.

\section{Discussion}

Neurological abnormalities in catatonia are well reported in the literature, but often under emphasized in clinical practice (Joseph et al., 1985; Freeman et al., 1975; Woods, 1980). They are important because they are markers of focal cerebral dysfunction, and thus may help in understanding the disordered physiology involved in catatonia.

Both the patients described here developed stuttering during the catatonic phase of a bipolar depression. It is known that lateralized cerebral functions, such as language, can be affected during some cases of acute psychosis and affective decompensation (Flor-Henry, 1983), and when stuttering occurs under these circumstances, it raises the possibility that in some catatonic bipolar patients it is due to an underlying disturbance of cerebral laterality.

Observations from these patients and other published data are consistent with the possibility and will be considered in turn.

Mr A stuttered while recovering from catatonia, Ms B while becoming catatonic. In each it occurred as part of a gradual transition between normal language and catatonic mutism. During the transition, varying degrees and types of language disorder were seen. Mr A initally had what is best described as a transcortical motor aphasia that changed to a transcortical sensory type during recovery. 
Ms B probably had a severe transcortical motor aphasia before becoming mute.

Arguably, their aphasias might be categorized as subcortical in the presence of so many basal ganglia signs. Several reports have appeared in which aphasia and stuttering occur with basal ganglia pathology (Damasio et al., 1982; Koller, 1983). Also their mutism was probably a severe endpoint form of aphasia.

Either the initiation or cessation of stuttering in adult life is considered a rare event (Helm et al., 1978). Transient acquired stuttering has been associated with focal left hemispheric pathology, and permanent acquired stuttering with bilateral pathology (Helm et al., 1978). In both types, there is frequent evidence of more generalized hemispheric damage, and varieties of aphasia and articulatory difficulties are commonly encountered (Helm et al., 1978). The two cases described here are thus typical of adults with acquired stuttering in both clinical presentation and location of demonstrated pathology.

The neurophysiology of stuttering is poorly understood, but it may occur when hemispheric language dominance is competitive or coequal (Helm-Estabrooks $e t$ $a l ., 1986)$. Thus any process that impairs the function of the language dominant hemisphere, so that its influence on the vocal expression of language becomes equal to that of the nondominant hemisphere, could cause acquired stuttering.

Affective decompensation could thus serve to alter the relationship between the language functions of the dominant and nondominant hemispheres and be permissive for transient stuttering. Affective disorders occur in some patients with disturbed hemispheric interactions (Flor-Henry, 1983).

The cases presented here are consistent with the hypothesis that in some bipolar patients affective decompensation, and the onset or resolution of catatonia, in some way compromises the relationship between the hemispheres in the control of language. Aphasia and mutism may be the result. In special circumstances, possibly of transient coequal hemispheric language dominance, transient stuttering could occur.

\section{References}

Damasio, A.R., Damasio H., Rizzo, M., et al. (1982). Aphasia with nonhemorrhagic lesions in the basal ganglia and internal capsule. Archives of Neurology, 39, 15-20.

Flor-Henry, P. (1983). "Cerebral Basis of Psychopathology". John Wright, Boston.

Freeman, J.M., Finkelstein, J.D. and Mudd, S.H. (1975). Folate - responsive homocystinuria and schizophrenia. New England Journal of Medicine, 292, 491-496.

Gelenberg, A.J. (1976). The catatonic syndrome. Lancet, 1, 1339-1341.

Helm, N.A., Butler, R.B. and Benson, D.F. (1978). Acquired stuttering. Neurology, 28, 1159-1165.

Helm-Estabrooks, N., Yeo, R., Geschwind, N., Freedman, M. and Weinstein, C. (1986). Disappearance and reappearance of stuttering with acquired brain lesions. Neurology, 36, 1109-1112.

Jenkyn, L.R., Walsh, D.B., Culver, C.M. and Reeves, A.G. (1977). Clinical signs in diffuse cerebral dysfunction. Journal of Neurology, Neurosurgery and Psychiatry, 40, 956-966.

Joseph, A.B., Anderson, W.H., O'Leary, D.H. (1985). Brainstem and vermis atrophy in catatonia. American Journal of Psychiatry, 142, 352-354.

Koller, W.C. (1983). Dysfluency (stuttering) in extrapyramidal disease. Archives of Neurology, 40, $175-177$. 
Lhermitte, F. (1983). "Utilization behavior" and its relation to lesions of the frontal labes. Brain, 106, 237-255.

Slater, E. and Roth, M. (1977). "Clinical Psychiatry", 3rd edn. Baillière Tindall, London.

Woods, S.W. (1980). Catatonia in a patient with subdural hematomas. American Journal of Psychiatry, 137, 983-984. 


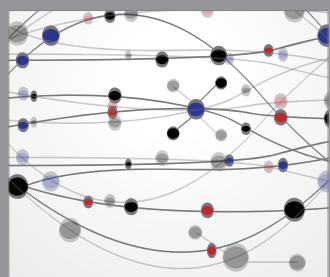

The Scientific World Journal
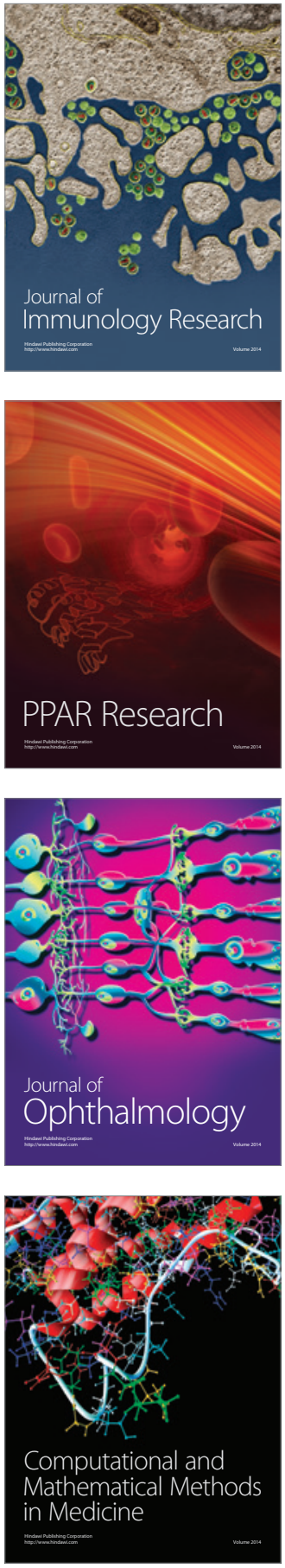

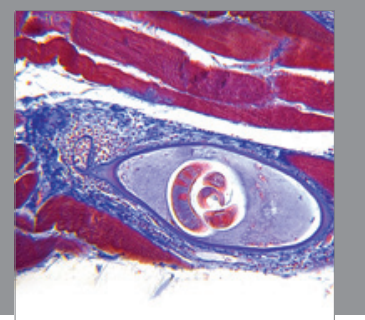

Gastroenterology

Research and Practice
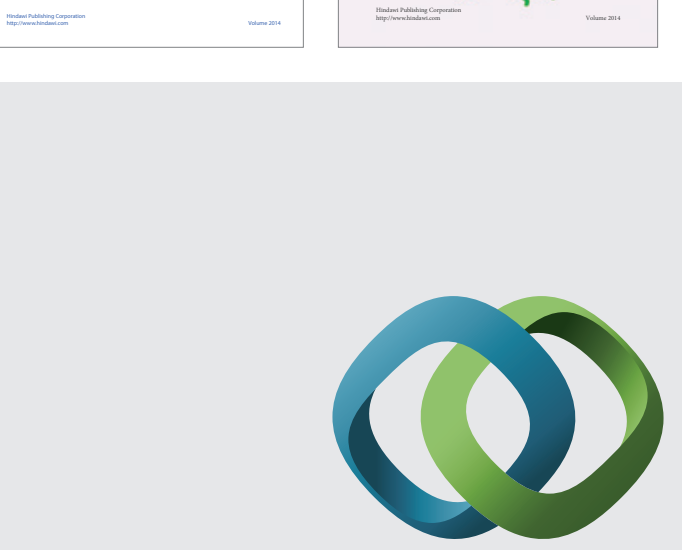

\section{Hindawi}

Submit your manuscripts at

http://www.hindawi.com
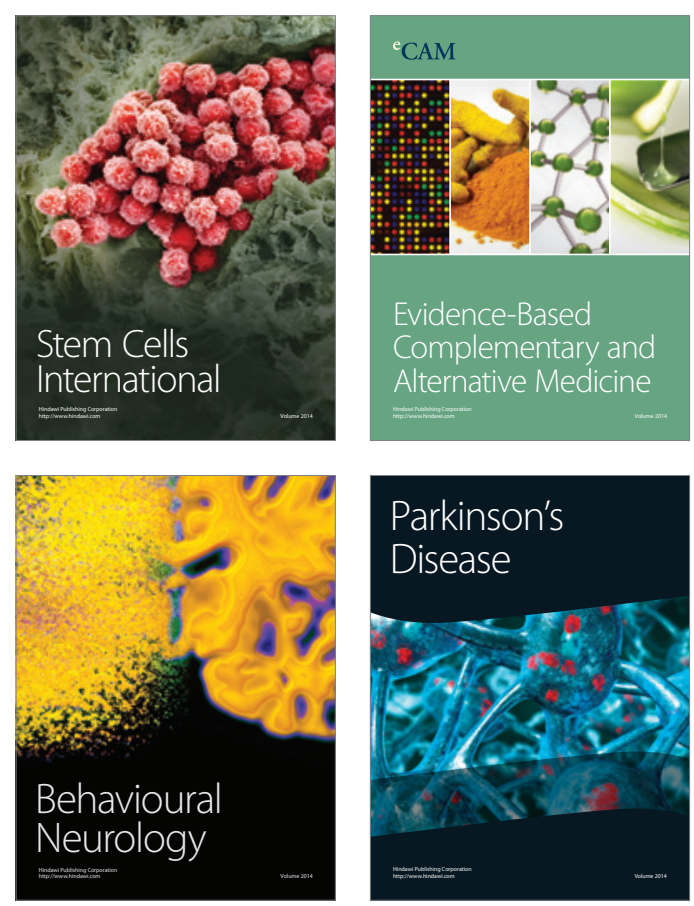

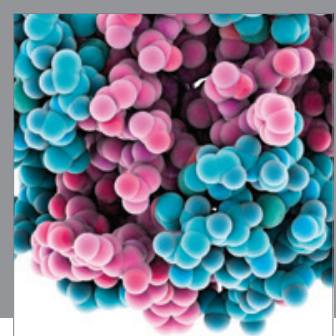

Journal of
Diabetes Research

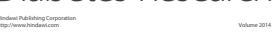

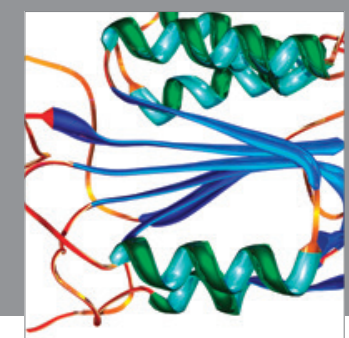

Disease Markers
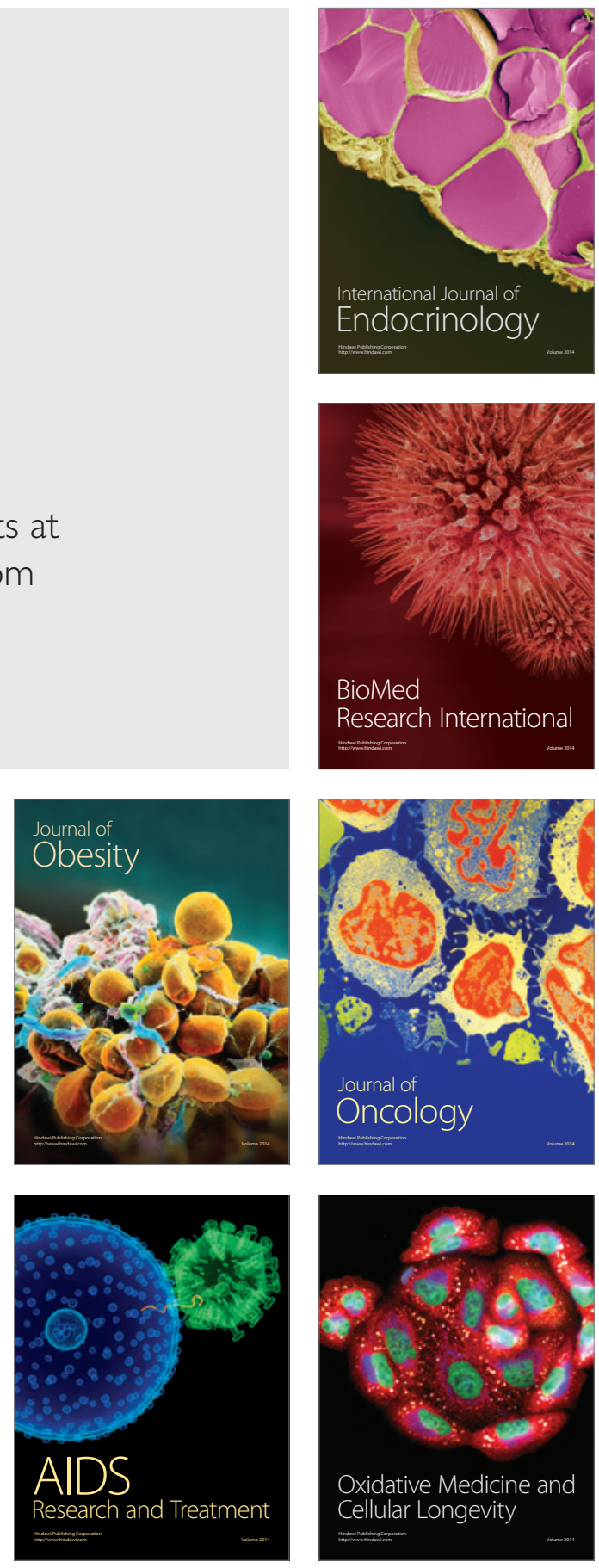